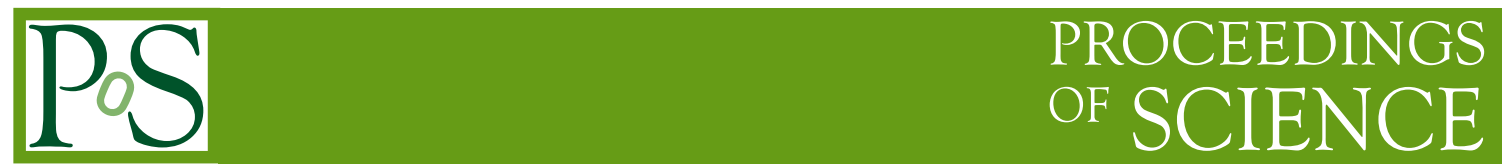

\title{
4U 0115+634: onset and evolution of a QPO
}

\section{Pere Blay *}

GACE - Image Processing Laboratory, University of Valencia

E-mail: pere.blay@uv.es

A detailed analysis of the BeX system $4 \mathrm{U} 0115+63$ is presented. ISGRI data of the last two bright outbusrts of the source (September, 2004 and April 2008) have been used. The overall behaviour of $4 \mathrm{U} 01156+63$ is that of a typical transient BeX system. However, ISGRI data show very interesting timing properties, and tell a beautiful story about the onset and evolution of a 2.1 $\mathrm{mHz}$ and a $1 \mathrm{mHz}$ QPOs, close in time to the maximum luminosity, in the 2004 and 2008 outbursts respectively. QPO changes and its possible origin are discussed.

8th INTEGRAL Workshop "The Restless Gamma-ray Universe"

September 27-30 2010

Dublin Castle, Dublin, Ireland

${ }^{*}$ The author aknowledges support from the spanish Ministerio de Ciencia e Innovacion through project 20100026ASIM. 


\section{Introduction.}

4U 0115+634 is a High Mass X-Ray Binary System (HMXRB), that is, a massive star and a compact star orbiting together around their common centre of mass. As a result of the mass transfer from the massive star onto the compact one, high energy is produced, i.e., X-rays and Gamma-rays are emitted. To be more concrete, the massive star in $4 \mathrm{U} 0115+634$ is a Be star. A Be star is a very peculiar type of star which is in its main sequence lifetime and forms an equatorial disk-like envelope, also called decretion disk. How this disk-like structure is formed is still an unknown fact, but it is believed to be linked to very rapid rotation and stellar pulsations. As a consequence of hosting a Be star, $4 \mathrm{U}$ 0115+63 is also grouped into the Be-X-Ray Binary systems type (BeXRB), which is a special case of HMXRB where the compact star interacts with the disk-like envelope around the Be star. On the other hand, the compact star in $4 \mathrm{U} 0115+634$ is a Neutron Star (NS). High energy emission is produced very close to the surface of the neutron star at its magnetic polar caps. It happens this way because the NS possesses an intense magnetic field. The matter pulled away from the Be disk by the NS (which is a plasma, and, therefore, it is highly ionized) will free-fall until it reaches the magnetosphere of the NS, i.e., the region where the magnetic field intensity becomes important and interacts with the falling matter. The downwards movement of the plasma is decelerated at this point and the plasma is driven along the magnetic field lines towards the magnetic polar caps of the NS. When the magnetic axis and the rotational axis of the NS are misaligned, this phenomenon results in the typical lighthouse-like emission of pulsars. We see the $\mathrm{X}$-ray emission once (or twice) each time the neutron star rotates.

In such systems, the number of different physical phenomena and its complexity are both great. We will concentrate only on a very particular aspect, which is the behaviour of the falling matter when approaching the NS magnetosphere. In BeXRB systems an accretion disk is very likely to be formed around the NS magnetosphere. This is a disk-like structure which the falling matter forms as it spirals downwards. In the inner region of the accretion disk, in the outskirts of the magnetosphere, is where we believe that Quasi Periodic Oscillations (QPOs) take place for a limited time span. We will review what QPOs are in the next section, but for now we will introduce briefly the behaviour of $4 \mathrm{U} 0115+63$ in the $20-60 \mathrm{keV}$ energy range.

As we see in the two plots of Figure 1, 4U 0115+63 is not detected as a persistent emitter, but shows very bright and long ( 2 months) outbursts associated with periods of big structural changes in the disk of the Be star. INTEGRAL/IBIS/ISGRI has observed 4U 0115+63 during two such events, during August-October 2004 (seen only partially, a bit before the outburst maximum flux, and later on during the outburst decay) and during February-May 2008 (this occasion, the coverage was much more complete), both are shown in left and right panels of Figure 1. Light curves with a binning of $20 \mathrm{~s}$ where extracted and corrected of doppler effects due to binary motion in order to investigate the properties of the QPOs.

\section{4U $0115+63$ and its QPOs}

As we have introduced in the previous section, QPO stands for Quasi Periodic Oscillation. This terms refers to an oscillation which can not be described by a sinusoidal variation or a coherent sum of sinusoidal variations. These oscillations will not appear as a single narrow peak in the 
Agust-October 2004 event

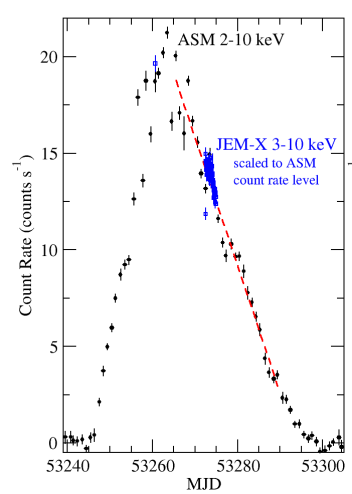

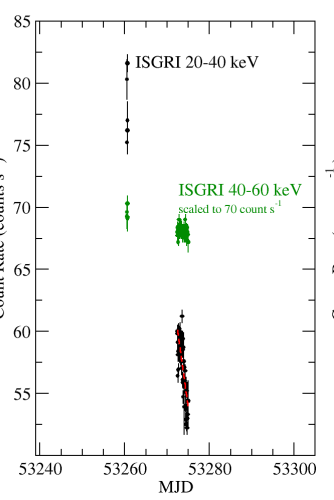

February-May 2008 event

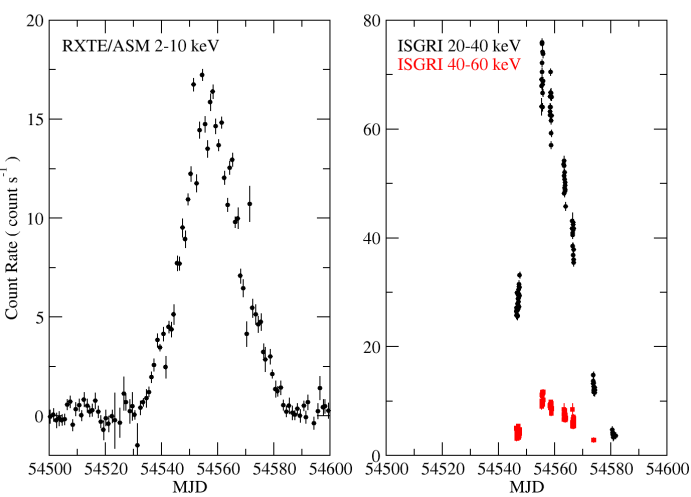

Figure 1: Left panel: Plots showing the evolution of the flux from $4 \mathrm{U} 0115+63$ in the $2-10 \mathrm{keV}$ (RXTE/ASM), 3-10 keV (INTEGRAL/JEM-X), 20-40 (INTEGRAL/ISGRI) and 40-60 keV (INTEGRAL/ISGRI) energy bands, during the outburst which took place in 2004. The red-dashed line represents the slope of the outburst decay in all cases. Right panel: Left panel: Plots showing the evolution of the flux from $4 \mathrm{U} 0115+63$ in the 2-10 keV (RXTE/ASM), 20-40 (INTEGRAL/ISGRI) and 40-60 keV (INTEGRAL/ISGRI) energy bands, during the outburst which took place in 2008.

fourier transform periodograms (or power spectra), but, instead, will show up as a broad feature which covers a wider range of frequencies.

In HMXRBs, QPOs are associated with the movement of the falling matter in the vicinity of the magnetosphere, i.e., in the inner parts of the accretion disk.

During the two outburst detected from $4 \mathrm{U} 0115+63$, the one which took place in 2004 and the one from 2008, a milihertz QPO is detected in the $20-40 \mathrm{keV}$ band. In both cases it happens close to the outburst peak, i.e., close to the maximum of emission. As we have stated before, the coverage of the outburst is much more complete in the 2008 event, however this will not affect the coverage of the QPOs, because of their very short duration and the fact that they were fortunately observed in both cases.

The $\sim 476$ s modulation of the 2004 QPO can be easily be seen in the $20 \mathrm{~s}$ binned light curves in the 20-40 keV energy range in Figure 2. Each plot corresponds to an INTEGRAL pointing of revolution 465, with time increasing upwards. Details of the evolution of the QPO can be seen on Figure 2. In the case of the 2008 QPO the modulation takes place on a time scale of $1000 \mathrm{~s}$ (see Figure 4 and 5). In the first case (2004) the duration of the QPO event is around $9000 \mathrm{~s}$, while in the latter case (2008) it lasts for about half a day.

The power spectra of the 3 consecutive INTEGRAL pointings of the 2004 outburst which show the $\sim 476$ s modulation (2nd, 3rd, and 4th plot of Figure 2, from bottom to top) are shown in Figure 3. There is a decrease in frequency of the QPO with time, the amplitude of the oscillation also being variable. The onset of the modulation is clearly visible in Figure 2, with no apparent modulation in the fist plot (counting from bottom) and showing an increasingly significant modulation in the following plots until reaching the 5th plot (at the top of the figure) where the modulation is not clearly seen any longer.

The 2008 QPO feature becomes clearly detectable during revolution 668, around the peak of 


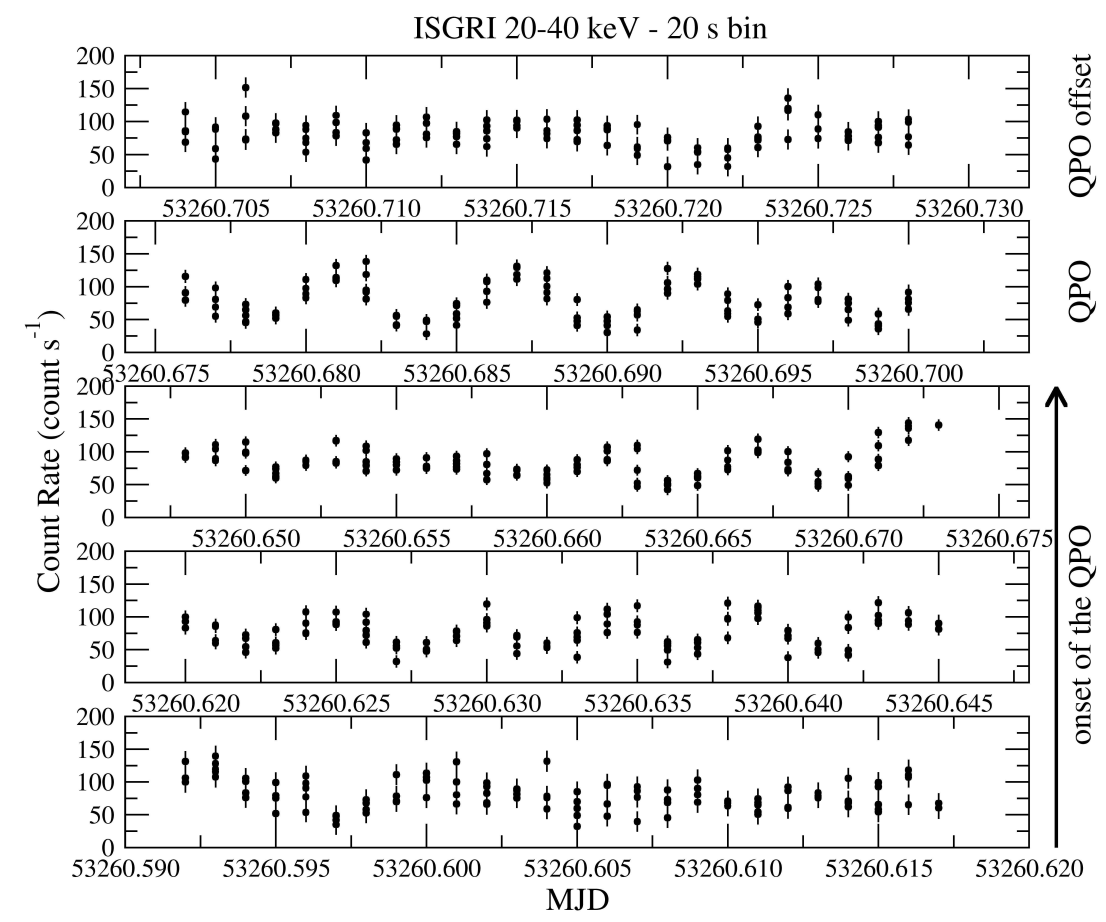

Figure 2: Light curves of 5 consecutive science windows during INTEGRAL revolution 465, close to the 2004 outburst peak. During the first 3 pointings (ordered in the upward direction) the modulation starts to be visible. The $\sim 476$ s modulation is clearly visible during the 4 th pointing and disappears in the latter one.
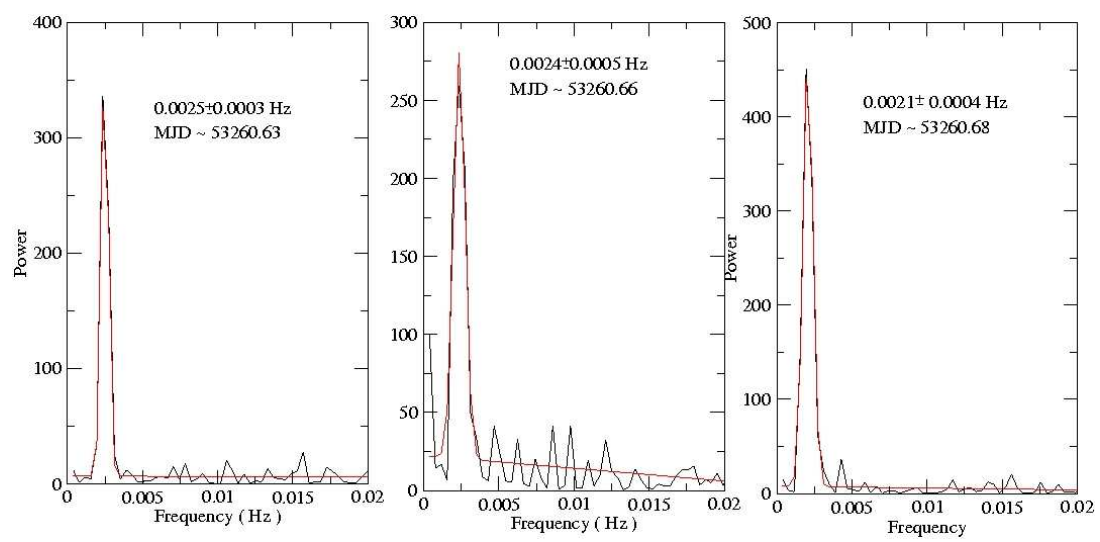

Figure 3: Evolution of the QPO during the second, third and fourth pointings shown in Figure 2, where time counts from bottom to top. 

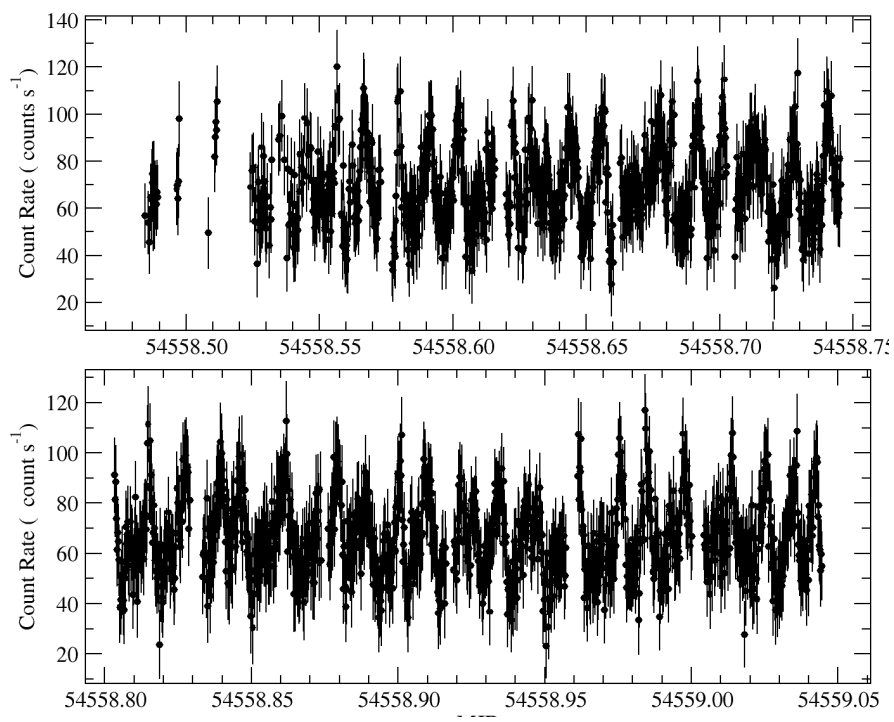

Figure 4: Detailed view of the $20-40 \mathrm{keV}$ light curve of $4 \mathrm{U} 0115+64$, binned to $20 \mathrm{~s}$, during INTEGRAL revolution 668 . A $\sim 1000 \mathrm{~s}$ modulation can be seen.

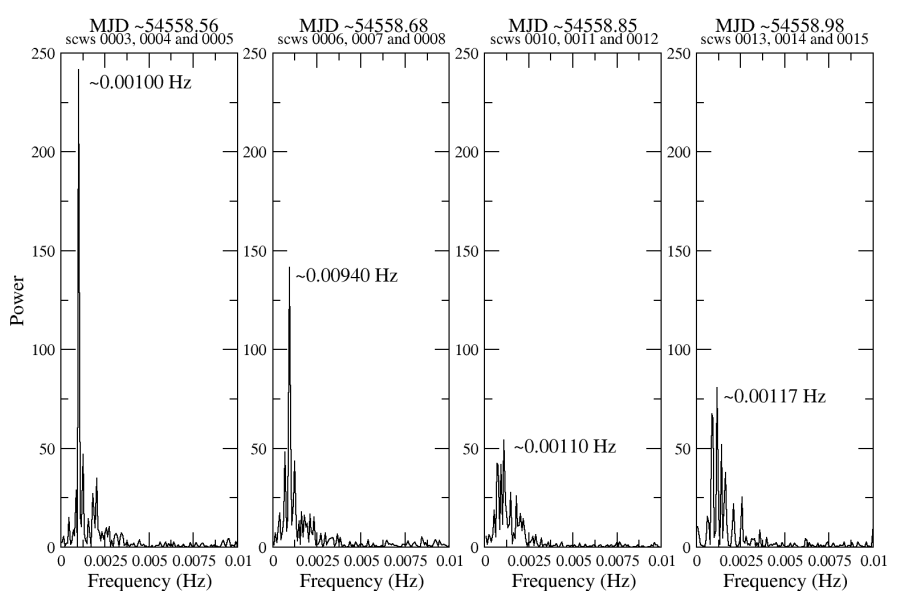

Figure 5: Power density spectra for sets of three consecutive pointings during INTEGRAL revolution 668. A $50 \mathrm{~s}$ binned light curve was used to derive the power spectra.

the outburst A detailed view of the $20-40 \mathrm{keV}$ light curve during revolution 668 is shown in Figure 4. The QPO feature disappears afterwards and it is not visible during revolution 669, already during the outburst decay. The longer time scale of the 2008 QPO implies that a more detailed analysis of the QPO evolution can be done by grouping several pointings. This is illustrated in Figure 5, where the power spectra for sets of three consecutive pointings of INTEGRAL revolution 668 are shown. The QPO feature is clearly variable, both in central frequency and in amplitude.

It is interesting to notice that the 2004 outburst had a steeper onset and a slower decay (as seen in the RXTE/ASM light curve in the Fgiure 1, in the first section of this poster), but this asymmetry is not present in the 2008 outburst, which seems to be quite symmetric instead. The peak flux is 
also higher in the 2004 outburst (above 80 counts s-1) than in the 2008 outburst (below 80 counts $\mathrm{s}-1)$. These small but significant differences may have an important effect in the rate at which mass is accreted by the neutron star. The accretion rate in both cases should be compared and it should be checked if that could be the origin of the difference in frequencies of the QPOs (the frequency of the 2008 QPO is half of that of the 2004 QPO, while in the 1999 outburst the frequency was similar to that in 2004 , i.e., $\sim 2 \mathrm{mHz}$, see Heindl et al, 1999)

\section{Conclusions}

There are several models dealing with the origin of QPOs, depending on the physical scenario under consideration. However we defend the simpler idea that QPOs must have a common physical mechanism related to the flow transition which matter suffers at the proximity of the NS magnetosphere (from a downwards movement in a spiral path at keplerian velocities, to a more sophisticated movement along the magnetic field lines onto the NS polar caps). In this transition region, due to the many interactions present, the number of instabilities and turbulences in the accreting flow must be high. It is likely that this instabilities and turbulences create quasi periodic perturbations which in some cases may couple in resonance leading to the observable QPO (see, for example, Mukhopadhyay, 2008).

If QPOs are linked to the interactions with the magnetosphere of the NS, and therefore with the spin changes of the NS, there must be a relation between spin-up rates and QPO frequencies. A more detailed follow-up of the pulse period evolution of $4 \mathrm{U} 0115+64$ along the outburst should be undertaken in order to investigate further this fact (see Mueller et al. 2010 for a detailed discussion about the pulse period evolution).

We have described the onset and evolution of two QPOs detected by INTEGRAL/ISGRI during two different outbursts of $4 \mathrm{U} 0115+63$ in the $20-60 \mathrm{keV}$ energy range. They are clearly visible in the 20-40 keV range, although both have a limited lifetime, which spans along the outburst peak in both cases. This fact must be an indication that the physical origin of both QPOs is similar, or at least there must be very similar physical conditions which make the QPO observable in both cases. However, the QPO central frequency has different values in both events. We plan to extend the analysis to lower energies in order to seek for the connection between the QPO frequency and the physical conditions of the accretion (accretion rate, spin-up rate, source luminosity, etc) in both outbursts.

\section{References}

[1] Heindl et al, 1999, ApJ, 521, L49

[2] Mueller S., 2010, PoS(INTEGRAL 2010)116

[3] Mukhopadhyay, B., 2008, AIP Conf. Proc., 1053, 343 